\title{
The Optimum Extraction Process for Radix glycyrrhizae and Angelica dahurica (Fisch.) Benth.et Hook with Orthogonal Design
}

\author{
Hong Duan*, Ke Feng Zhai*, Wen Gen Cao, Gui Zhen Gao, Ling Ling Shan, Liang Zhao \\ Engineering Research Center of Natural Medicine and Functional Food, Institute of Pharmaceutical Biotechnology, School of Biological \\ and Food Engineering, Engineering Research Center of Special Farm Seed Production, Suzhou University, 49, Bianhe Road, Suzhou, \\ CHINA.
}

\begin{abstract}
Background: To develop the optimal extractive technique of the Radix glycyrrhizae and Angelica dahurica (Fisch.) Benth.et Hook in the Fengshiding dropping pill. Methods: Single factor experiments were carried out on the effect of water-ethanol extraction by the multiple guidelines grading of the dried extract quantity, the contents of liquiritin and imperatorin. Furthermore, orthogonal experimental design was used for the optimization of cross-functional process. The dried extract quantity and the contents of liquiritin and imperatorin were used as targets. The interaction among solid-liquid ratio, ethanol concentration, extraction time and extraction times was investigated by the analysis of variance. Data processing was carried out with the multiple guidelines grading method for optimizing the extraction condition. Results: The optimal extracting condition was founded to be successively 3 decoctions with 12 times of $75 \%$ alcohol for $1 \mathrm{~h}$. Conclusion: The optimal process is stable and feasible.
\end{abstract}

Key words: Radix glycyrrhizae, Angelica dahurica (Fisch.) Benth.et Hook, Liquiritin, Imperatorin, Orthogonal test.

\section{INTRODUCTION}

The prescription of the Fengshiding dropping pill comes from the Fengshiding tablet that exists in preparation of Chinese medicine in twelfth volumes from drug standard of ministry of public health (Standard No. WS3-B2297-97). The Fengshiding tablet is a traditional Chinese preparation and made up by Alangium chinense, Cynanchum paniculatum, Radix ghycyrrbizae and Angelica daburica (Fisch.) Benth.et Hook. ${ }^{1}$ It has good effect on promoting blood circulation and analgesia in the anti-rheumatic arthritis or anti-rheumatoid arthritis, ${ }^{1}$ which has been for clinical practice for many years with definite therapeutic effects. However, Angelica daburica powder can be directly used as medicine in the Fengshiding tablet, which leads to low content of digestion components and high daily doses in vivo. In order to improve patient compliance and the preparation quality, the formulation and preparation of the Fengshiding tablet need to be re-optimized.

Recent studies have shown that licorice extract and its bioactive component liquiritin can be applied for the treatment of inflammationrelated disorders, such as oxidative liver damage and inflammation diseases; ${ }^{2}$ Angelica daburica extract $^{3}$ and its bioactive component imperatorin $^{4}$ can be applied for the anti-inflammatory effects and the antiallergic effects. Therefore, the representative extract of liquiritin and imperatorin lays an anti-inflammatory medicinal material foundation for development of the Fengshiding dropping pill. In addition, several methods, including organic solvent extraction, microwave-assisted extraction, ${ }^{5}$ and ultrasonicassisted extraction (UAE), ${ }^{6}$ have been widely employed for the extraction of single herb. However, few reports have focused on combination extraction process of Radix glycyrrbizae
Submission Date: 15-04-2017; Revision Date: 22-05-2017; Accepted Date: 17-07-2017

DOI: 10.5530/ijper.51.4s.92 Correspondence: Dr. Hong Duan,

Engineering Research Center of Natural Medicine and Functional Food, Institute of Pharmaceutical Biotechnology, School of Biological and Food Engineering, Engineering Research Center of Special Farm

Seed Production, Suzhou University, 49, Bianhe Road, Suzhou, 234000, CHINA.

Phone no: 86-557-2871681 E-mail: szxydh@163.com

Dr. Ke-Feng Zhai,

Engineering Research

Center of Natural Medicine and Functional Food, Institute of Pharmaceutical Biotechnology, School of Biological and Food Engineering, Engineering Research Center of Special Farm

Seed Production, Suzhou University, 49, Bianhe Road, Suzhou, 234000, CHINA.

Fax and Tel: 86-557-2871037

E-mail: kefengzhai@163.com

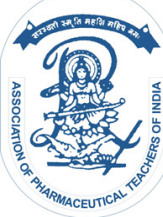

www.ijper.org 
and Angelica dahurica (Fisch.) Benth.et Hook for liquiritin and imperatorin.

In the present study, based on the common chemical properties of the representative extract of liquiritin and imperatorin (structure shown in Figure 1), we chose ethanol-water combination extraction of liquiritin ${ }^{2,7,8}$ and imperatorin ${ }^{9,10,11}$ from Radix glycyrrbizae and Angelica daburica (Fisch.) Benth. et Hook according to similarity-intermiscibility theory. In order to estimate and optimize the factors affecting extraction to achieve maximum recovery, we investigated the effect of water-ethanol extraction on the multiple guidelines grading of the dried extract quantity, the contents of liquiritin and imperatorin by single factor experiment. A secondorder polynomial model was set up to predict the acquired the multiple guidelines grading using orthogonal test. The interaction among solid-liquid ratio, ethanol concentration, extraction time and extraction times was investigated by the analysis of variance. Finally, the optimal extraction condition was obtained and provided a basis for new drug development of the Fengshiding dropping pill.

\section{MATERIALS AND METHODS}

\section{Materials}

The medicinal materials of Radix glycyrrbizae and Angelica daburica (Fisch.) Benth.et Hook were purchased from the Kangyuan Pharmaceutical Co. Ltd. in Anhui province, China. The Control products of liquiritin (batch code: 111610-200604) and imperatorin (batch code: 110826200712) were purchased from China Materia Medica Biological Product Inspection Institute. Methanol was chromatographic grade and the other chemicals were of analytical grade.

\section{Extraction process of liquiritin and imperatorin}

Ten $\mathrm{g}$ of mixture medicinal material (prescription proportion: Radix glycyrrbizae: Angelica daburica=2.5:1, $\mathrm{w} / \mathrm{w}$ ) was blended with complex solvent of ethanol and water. The values of solid-liquid ratio, ethanol concentration, extraction time, and extraction times were set according to the requirement of experiment. The extract was filtered using a sand core funnel, and then fixed to $250 \mathrm{~mL}$ with the same extracting solution. The physic liquor $10 \mu \mathrm{L}$ was precisely sampled to determination of the contents of liquiritin and imperatorin by HPLC (LC-20AT, Shimadzu Corporation, Japan) and $25 \mathrm{~mL}$ was precisely sampled to determination of the dried extract quantity. The dried extract quantity was calculated using the following equation:

The dried extract quantity $=$ weight of dried $25 \mathrm{~mL}$ physic liquor $\times 250 / 25 / 10 \times 100 \%$

\section{Determination method of liquiritin and imperatorin}

The determination was carried out over a Shim-pack VP-ODS $C_{18}$ column $(250 \times 4.6,5 \mu \mathrm{m})$ at the temperature of $30^{\circ} \mathrm{C}$. The mobile phase ${ }^{1}$ consisted of methanol (A) - water (B) with the gradient elution (0-3 min, 45\% A; 3-6 min, 55\% A; 6-8 min, 65\% A; 8-50 min, 65\% A). The flow rate: $0.6 \mathrm{~mL} / \mathrm{min}$, and detection wavelength was set at $276 \mathrm{~nm}$ (detecting liquiritin) and $300 \mathrm{~nm}$ (detecting imperatorin). Figure 2 shows HPLC chromatograms of determination.

Preparation of reference substance: precisely sampled the standard liquiritin $2.67 \mathrm{mg}$ and imperatorin $3.66 \mathrm{mg}$ into $25 \mathrm{~mL}$ capacity bottle, ultrasonic dissolving in appropriate methanol, added methanol to scale, and shook up.

The establishment of standard curve: precisely transfered $1,2,34,5 \mathrm{~mL}$ the solution to $10 \mathrm{~mL}$ capacity bottle, added methanol to scale, shook up, and determined it at $276 \mathrm{~nm}$ and $300 \mathrm{~nm}$ wavelength at the same time. The regression equations were obtained as $Y=25094 X-6154.2(r=0.9997, n=5$, liquiritin $)$ and $Y=86702 X+29706 \quad(r=0.9996, n=5$, imperatorin $)$. The linear ranges were $10.68-53.40 \mu \mathrm{g} / \mathrm{mL}$ for liquiritin and $14.64-73.20$ and $\mathrm{g} / \mathrm{mL}$ for imperatorin. This method was simple, and had good linear relationship.

\section{Experimental design}

\section{Single factor experiments ${ }^{12}$}

Active components were extracted using complex solvent of ethanol and water in boiling status. The
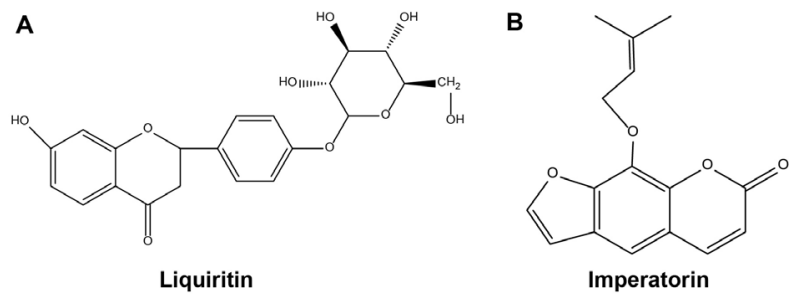

Figure 1: Chemical structures. (A) Chemical structure of liquiritin (B) Chemical structure of imperatorin.
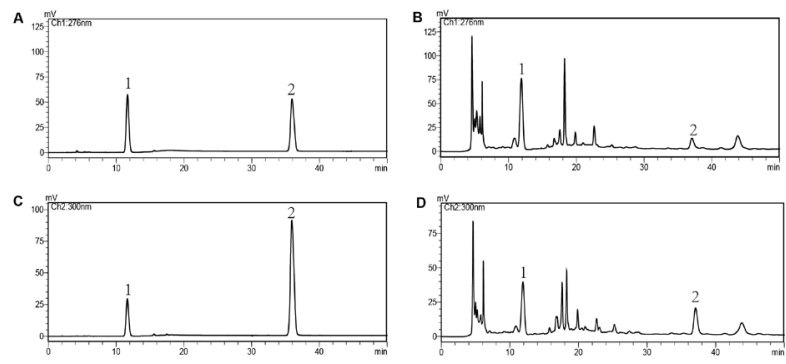

Figure 2: HPLC chromatograms of reference substance (A) and sample (B) at $276 \mathrm{~nm}$, and of reference substance(C) and sample (D) at $300 \mathrm{~nm}$ 1-liquiritin 2-imperatorin. 
proportion of ethanol in the solvent varied in the range of $55-95 \%(\mathrm{v} / \mathrm{v})$. The best solvent concentration was selected according to the value of overall desirability (OD). The value of $\mathrm{OD}$ was calculated using the following equation: $\mathrm{OD}=$ (liquiritin content/ the maximum of liquiritin content $) \times 0.4$ + (imperatorin content / the maximum of imperatorin content $) \times 0.4+$ (extract rate / the maximum of extract rate) $\times 0.2$.

Active components were extracted using the best solvent selected in the previous step. The solid-liquid ratio varied from 1:8 to $1: 16$ while fixing the extraction time constant at $1 \mathrm{~h}$.

Using the best ethanol concentration selected in first step, active components were extracted during various extraction time course ranges from 0.5 to $4 \mathrm{~h}$ at the optimum solid-liquid ratio determined in the second step. Using the best ethanol concentration selected in first step and the optimum solid-liquid ratio determined in the second step, active components were extracted during various extraction times from 1 to 5 at the optimum extraction time determined in the third step.

The ranges of three factors were determined for $\mathrm{L}_{9}\left(3^{4}\right)$ orthogonal test according to the results of experiments.

\section{Experiment of $L_{9}\left(3^{4}\right)$ orthogonal test ${ }^{13}$}

Optimization of extracting condition was carried out using $\mathrm{L}_{9}\left(3^{4}\right)$ orthogonal test, which was consisted of 9 experimental runs and used as visual analysis and variance analysis for optimization.

\section{Verification of the optimal conditions}

Optimal conditions for active components extraction were obtained using the variance analysis model of orthogonal test. The practical acquired ratio was obtained under the optimal conditions. The acquired OD of experimental and predicted was compared in order to determine the validity of the model.

\section{RESULTS}

\section{Results of single factor experiment}

As can be seen from Figure 3A, the acquired OD as a function of ethanol concentration followed a parabola shape. The acquired OD increased with increasing of the ethanol proportion in the extraction medium up to $85 \%$ and then began to decline with the further increase of ethanol proportion in the extraction medium. As it was shown in Figure 3B, the acquired OD was increased gradually with increasing of the solid-liquid ratio and the maximum acquired OD of $99.14 \%$ was obtained when the solid-liquid ratio was 1:14. As observed in Figure 3C,

\begin{tabular}{|c|c|c|c|}
\hline \multirow[b]{2}{*}{ Level } & & Factors & \\
\hline & $\begin{array}{c}\text { A ethanol } \\
\text { concentration (\%) }\end{array}$ & $\begin{array}{l}\text { B solid-liquid } \\
\text { ratio (times) }\end{array}$ & $\begin{array}{l}\text { C extraction } \\
\text { time (h) }\end{array}$ \\
\hline 1 & 65 & 12 & 1.0 \\
\hline 2 & 75 & 14 & 2.0 \\
\hline 3 & 85 & 16 & 3.0 \\
\hline
\end{tabular}
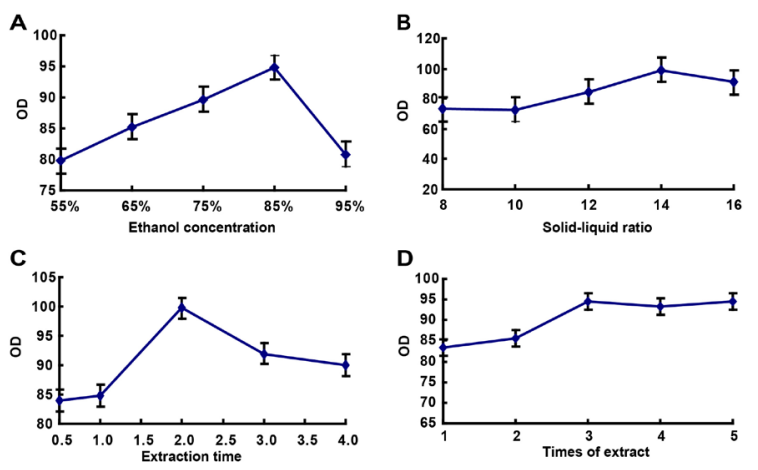

Figure 3: Results of single-factor experiments (A) ethanol concentration (B) solid-liquid ratio (C) extraction time (D) extraction times.

the acquired $\mathrm{OD}$ as a function of extraction time followed a parabola shape. The acquired OD increased with increasing of the extraction time in the extraction medium up to $2 \mathrm{~h}$ and then began to decline with the further increase of extraction time in the extraction medium. As shown in Figure 3D, the acquired OD was increased slightly with increasing of the extraction times. This observation suggested that the extraction times had no significant effect on the acquired value of $\mathrm{OD}$ and fixed it on 3 times.

\section{Results of orthogonal test}

Based on the single-factor experiments, the following values influencing the acquired OD were selected as the ranges of independent variables. The levels of factors were shown in Table 1. The design arrangement and the experimental results were shown in Table 2. Analysis of variance was summarized in Table 3 . As visual analysis shown in Table 2, the main influence factors on OD from big to small in proper order are A, B, and C. The $F$-test and $p$ value in Table 3 indicated that ethanol concentration $(A)$ had the largest effect on OD $(P<0.05)$, and the others had no significance on OD. Considering cost reduction and energy saving, the optimal extracting condition was founded to be successively 3 decoctions with 12 times of $75 \%$ alcohol for $1 \mathrm{~h}$. 


\begin{tabular}{|c|c|c|c|c|c|c|c|c|}
\hline No. & A & B & C & D & $\begin{array}{l}\text { extract } \\
\text { rate/\% }\end{array}$ & $\begin{array}{c}\text { liquiritin } \\
\text { content/mg/g }\end{array}$ & $\begin{array}{l}\text { imperatorin } \\
\text { content } / \mathrm{mg} / \mathrm{g}\end{array}$ & OD \\
\hline 1 & 1 & 1 & 1 & 1 & 22.73 & 1.6719 & 0.2620 & 90.74 \\
\hline 2 & 1 & 2 & 2 & 2 & 20.11 & 1.7740 & 0.2959 & 95.12 \\
\hline 3 & 1 & 3 & 3 & 3 & 20.86 & 1.6609 & 0.3032 & 94.30 \\
\hline 4 & 2 & 1 & 2 & 3 & 18.57 & 1.7093 & 0.2863 & 91.09 \\
\hline 5 & 2 & 2 & 3 & 1 & 21.88 & 1.8485 & 0.2978 & 98.54 \\
\hline 6 & 2 & 3 & 1 & 2 & 18.00 & 1.7147 & 0.2968 & 92.09 \\
\hline 7 & 3 & 1 & 3 & 2 & 18.63 & 1.5415 & 0.2487 & 82.56 \\
\hline 8 & 3 & 2 & 1 & 3 & 20.24 & 1.5722 & 0.2788 & 88.61 \\
\hline 9 & 3 & 3 & 2 & 1 & 18.11 & 1.5865 & 0.2554 & 83.96 \\
\hline $\mathrm{K}_{1}$ & 93.387 & 88.130 & 90.480 & 91.080 & & & & \\
\hline $\mathrm{K}_{2}$ & 93.907 & 94.090 & 90.057 & 89.923 & & & & \\
\hline $\mathrm{K}_{3}$ & 85.043 & 90.117 & 91.800 & 91.333 & & & & \\
\hline $\mathrm{R}$ & 8.864 & 5.960 & 1.743 & 1.410 & & & & \\
\hline
\end{tabular}

\begin{tabular}{|c|c|c|c|c|c|}
\hline \multicolumn{7}{|c|}{ Table 3: Analysis of variance. } \\
\hline factor & sum of square & freedom & $\boldsymbol{F}$ value & $\boldsymbol{F}$ critical value & significance \\
\hline A & 148.440 & 2 & 43.788 & 19.000 & $P<0.05$ \\
\hline B & 55.256 & 2 & 16.300 & 19.000 & \\
\hline C & 4.961 & 2 & 1.463 & 19.000 & \\
\hline error & 3.39 & 2 & & & \\
\hline
\end{tabular}

$F_{0.1}(2,2)=9.00 F_{0.05}(2,2)=19.00 \quad F_{0.01}(2,2)=99.00$

\section{Verification of the results}

The suitability of OD was tested using the recommended optimal conditions. The experimental values $(96.62,97.51,95.00, n=3)$ were found to be close to the predicted one.

\section{DISCUSSION}

The Fengshiding tablet, a traditional Chinese preparation, is composed of Alangium chinense, Cynanchum paniculatum, Radix Glycyrrbizae and Angelica Daburica (Fisch.) Benth.et Hook. ${ }^{1}$ However, the Fengshiding tablet has many defects such as the indistinctness of its effective substances, low content of digestion component, high daily doses, poor stability and quality control. In order to improve patient compliance and the preparation quality, the formulation and preparation of the Fengshiding tablet need to be re-optimized. Following the original preparation functions, we must fully consider the characteristics and the roles of each medicinal material in the prescription, when the preparation is improved. On the basis of the latest achievements of modern pharmacology research, we made clear its effective components and extracts. The combination extract taken liquiritin and imperatorin as representatives from Radix glycyrrbizae and Angelica daburica (Fisch.) Benth.et Hook, which showed significant activity of anti-inflammatory and analgesic, ${ }^{14,15,16}$ played an important role in the Fengshiding dropping pill. Furthermore, recent studies have indicated that the extraction of salicin ${ }^{17,18,19}$ from Alangium chinense has the antinociceptive and anti-inflammatory activity, and steam distillation of paeonol ${ }^{20,21,22}$ from Cynanchum paniculatum has suppressed swelling, inflammatory reaction and cell impairment. The new extraction procedure for Alangium chinense or Cynanchum paniculatum in the Fengshiding dropping pill is definitely worth study in the future.

In the present study, we focused on the optimal extractive technique of the Radix glycyrrbizae and Angelica daburica (Fisch.) Benth.et Hook in the Fengshiding dropping pill. The dried extract quantity and the contents of liquiritin and imperatorin used as targets, we employed single factor experiments combined with orthogonal experimental design to improve the preparation process. Our results demonstrated that the optimal process for radix glycyrrbizae and angelica daburica (Fisch.) Benth.et Hook was stable and feasible, which could be applied in 
the future drug development of the Fengshiding dropping pill.

Of note, to find an easy way to analyse the speciments, we had tried a gradient solvent system (water, methanol and acetonitrile). ${ }^{1}$ The final choice of mobile phase was methanol-water, which can effectively separate the two markers (liquiritin and imperatorin) simultaneously. This mobile phase was relatively simple to operate and less harmful to the chromatographic column and instrument. In order to further shorten the analytical time and improve the sensitiv ity of analysis results, gradient elution was carried out with adopting double wavelength for determination of two kinds of ingredients simultaneously. It had been proved that the experimental establishment of the chromatographic conditions had got a good segregative result.

\section{CONCLUSION}

The optimum extraction process for Radix glycyrrbizae and Angelica daburica (Fisch.) Benth.et Hook in Fengshiding dropping pill was as follow: successively 3 decoctions with 12 times of $75 \%$ alcohol for $1 \mathrm{~h}$. Our verification of the results demonstrated that the optimal process for Radix glycyrrbizae and Angelica daburica (Fisch.) Benth.et Hook was stable and feasible. The precise combination extract of Radix glycyrrbizae and Angelica dahurica (Fisch.) Benth.et Hook laid a solid medicinal material foundation for new drug development of the Fengshiding dropping pill.

\section{ACKNOWLEDGMENT}

This research work was supported by the National Natural Science Foundation of China (No.81601034), the Natural Science Foundation Committee of Anhui Province (No.1608085QH185), the Key Natural Science Foundation of Anhui Educational Committee (No. KJ2015A220), the Key Project Fund for Outstanding Young Talents in Anhui Higher Education Institutions (No.gxyqZD2016348), and the Open Project Program of the Engineering Research Center of Special Farm Seed Production (No.2015YKF03, 2015YKF06, 2015YKF01) and Suzhou Regional Development Collaborative Innovation Center (No. 2016szxt03, 2015SZXTZXKFYB03).

\section{CONFLICT OF INTEREST}

The authors declare that they have no competing interests.

\section{ABBREVIATION USED}

UAE: Ultrasonicassisted extraction; OD: Overall desirability; HPLC: High performance liquid chromatography.

\section{REFERENCES}

1. Zhai KF, GAO GZ, CAO WG, Zhao L, Fang XM, Duan H. Simultaneous HPLC Determination of Four Active Compounds in Fengshiding Capsules, a Chinese Medicine. Indian J Pharm Sci. 2014;76(5):445-9.

2. Tian M, Bi W, Row KH. Solid-phase extraction of liquiritin and glycyrrhizic acid from licorice using ionic liquid-based silica sorbent. J Sep Sci. 2009;32(23-24):4033-9.

3. Yu JY, Ha JY, Kim KM, Jung YS, Jung JC, Oh S. Anti-Inflammatory activities of licorice extract and its active compounds, glycyrrhizic acid, liquiritin and liquiritigenin, in BV2 cells and mice liver. Molecules. 2015;20(7):13041-54.

4. Zhang X, Song Y, Han X, Feng L, Wang R, Zhang M, et al. Liquiritin attenuates advanced glycation end products-induced endothelial dysfunction via RAGE/ NF-KB pathway in human umbilical vein endothelial cells. Mol Cell Biochem. 2013;374(1-2):191-201.

5. Budzynska B, Boguszewska-Czubara A, Kruk-Slomka M, Skalicka-Wozniak K, Michalak A, Musik I, et al. Effects of imperatorin on scopolamine-induced cognitive impairment and oxidative stress in mice. Psychopharmacology. 2015;232(5):931-42.

6. Cao Y, Zhang Y, Wang N, He L. Antioxidant effect of imperatorin from Angelica dahurica in hypertension via inhibiting NADPH oxidase activation and MAPK pathway. J Am Soc Hypertens. 2014;8(8):527-36.

7. Geng Y, Zhu S, Lu Z, Xu H, Shi JS, Xu ZH. Anti-inflammatory activity of mycelial extracts from medicinal mushrooms. Int $\mathrm{J}$ Med Mushrooms. 2014;16(4):319-25.

8. Khayyal MT, El-Ghazaly MA, Abdallah DM, Okpanyi SN, Kelber O, Weiser D. Mechanisms involved in the anti-inflammatory effect of a standardized willow bark extract. Arzneimittel-Forschung. 2005;55(11):677-87.

9. Li Y, Wu Q, Deng Y, Lv H, Qiu J, Chi G, et al. D(-)-Salicin inhibits the LPS-induced inflammation in RAW264.7 cells and mouse models. Int Immunopharmacol. 2015;26(2):286-94.

10. Deng C, Yao N, Wang B, Zhang X. Development of microwave-assisted extraction followed by headspace single-drop microextraction for fast determination of paeonol in traditional Chinese medicines. J Chromatogr A. 2006;1103(1):15-21.

11. Zhai KF, Duan H, Luo L, Cao WG, Han FK, Shan LL, et al. Protective effects of paeonol on inflammatory response in IL- $1 \beta$-induced human fibroblast-like synoviocytes and rheumatoid arthritis progression via modulating NF-KB pathway. Inflammopharmacology. 2017; 25(5):523-32.

12. Krzywinski M, Altman N. Points of significance: Designing comparative experiments. Nat Methods. 2014;11(6):597-8.

13. Chen CJ, Zhao PC, Huang YM, Tong ZF, Li ZX. Preparation and characterization of activated carbon from eucalyptus sawdust I. activated by $\mathrm{NaOH}$. J Inorg Organomet Polym Mater. 2013;23(5):1201-9.

14. Al-Okbi SY. Nutraceuticals of anti-inflammatory activity as complementary therapy for rheumatoid arthritis. Toxicol Ind Health. 2014;30(8):738-49.

15. Huang GJ, Deng JS, Liao JC, Hou WC, Wang SY, Sung PJ, et al. Inducible nitric oxide synthase and cyclooxygenase-2 participate in anti-inflammatory activity of imperatorin from Glehnia littoralis. J Agric Food Chem. 2012;60(7):1673-81.

16. Chang YX, Zhang QH, Li J, Zhang L, Guo XR, He J, et al. Simultaneous determination of scopoletin, psoralen, bergapten, xanthotoxin, columbianetin acetate, imperatorin, osthole and isoimperatorin in rat plasma by LC-MS/MS for pharmacokinetic studies following oral administration of Radix Angelicae Pubescentis extract. J Pharm Biomed Anal. 2013;77:71-5.

17. Zahan R, Nahar L, Nesa ML. Antinociceptive and anti-inflammatory activities of flower (Alangium salvifolium) extract. Pak J Biol Sci. 2013;16(19):1040-5.

18. Khayyal MT, El-Ghazaly MA, Abdallah DM, Okpanyi SN, Kelber O, Weiser D. Mechanisms involved in the anti-inflammatory effect of a standardized willow bark extract. Arzneimittel-Forschung. 2005; 55(11): 677-87.

19. Li Y, Wu Q, Deng Y, Lv H, Qiu J, Chi G, et al. D(-)-Salicin inhibits the LPS-induced inflammation in RAW264.7 cells and mouse models. Int Immunopharmacol. 2015; 26(2):286-94. 
20. 20. Wang S, Qu W, Li T, Guo K, Liu W, Wang Z, et al. Xiangqing anodyne spray (XQAS): a combination of ethanol extracts of Cynanchum paniculatumand Illicium henryi for treating soft-tissue injury. Int J Clin Exp Med. 2015; 8(8):12716-25.

21. 21. Deng C, Yao N, Wang B, Zhang X. Development of microwave-assisted extraction followed by headspace single-drop microextraction for fast determination of paeonol in traditional Chinese medicines. J Chromatogr A. 2006; 1103(1): 15-21.

22. 22. Zhang $L$, Tao $L$, Shi $T$, Zhang $F$, Sheng $X$, Cao $Y$, et al. Paeonol inhibits B16F10 melanoma metastasis In vitro and In vivo via disrupting proinflammatory cytokines-mediated NF-kB and STAT3 pathways. IUBMB Life. 2015; 67(10):778-88

\section{PICTORIAL ABSTRACT}
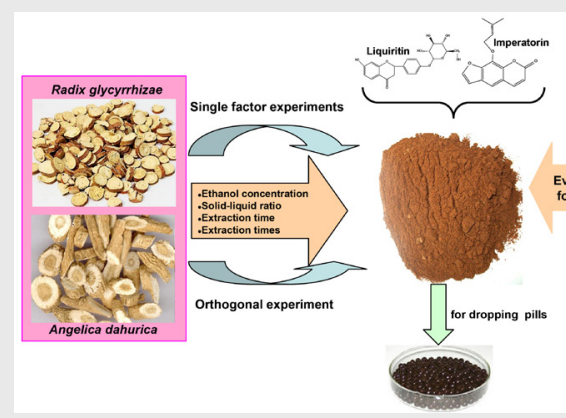

\section{SUMMARY}

- A validated method for estimation of liquiritin and imperatorin in the ethanol-water combination extraction from Radix glycyrrbizae and Angelica dahurica (Fisch.) Benth.et Hook through RP-HPLC.

- Data processing was carried out with the multiple guidelines grading method for optimizing the extraction condition.

- Single factor experiments and orthogonal test were carried out to develop the optimal extractive technique of the Radix glycyrrbizae and Angelica daburica (Fisch.) Benth.et Hook in the Fengshiding dropping pill.

- The optimal extracting condition was founded to be successively three decoctions with 12 times of $75 \%$ alcohol for $1 \mathrm{~h}$. This extracting process was stable and feasible and provided a basis for new drug development of the Fengshiding dropping pill.

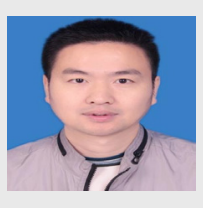

Mrs. H. Duan: She is an Assistant Professor having 13 years experience in teaching whose main interests lies in screening different plant extracts and their isolates for their potential pharmacological activity.

Dr. K. F. Zhai: He is working as an Associate Professor, in the School of Biological and Food Engineering at Suzhou University, Suzhou. He obtained his Ph.D degree in Pharmacy from China Pharmaceutical University, Nanjing. Dr. K. F. Zhai has research experience in the area of Pharmacy comprising Screening different plant extracts, Evaluating their potential pharmacological activity and Drug discovery.

Cite this article: Duan H, Zhai KF, Cao WG, Gao GZ, Shan LL, Zhao L. The Optimum Extraction Process for Radix glycyrrhizae and Angelica dahurica (Fisch.) Benth.et Hook with Orthogonal Design. Indian J of Pharmaceutical Education and Research. 2017;51(4S):S631-S6. 